\title{
Estimation of the remanufacturing cost of engineering machinery hydraulic cylinder
}

\author{
Pin-pin Qin ${ }^{1}$ \\ ${ }^{1}$ College of Mechanical Engineering, Guangxi \\ University, \\ Nanning 530004, China \\ Jian-wei Shu ${ }^{1}$ \\ ${ }^{1}$ College of Mechanical Engineering, Guangxi \\ University, \\ Nanning 530004, China
}

\begin{abstract}
The remanufacturing cost of estimation, the accuracy of the estimates and the time it takes to produce cost estimates are important factors to remanufacturing organizations. This paper constructed and trained a back-propagation (BP) neural network to forecast the remanufacturing cost of engineering machinery hydraulic cylinder. According to hydraulic cylinder remanufacturing process, 11 cost-related features have been identified and quantified. Neural networks prediction model was set up with features as input and the cost as the output. The network was trained and validated using 15 samples. The testing results on another 1 sample are promising, which the prediction errors were in the acceptable range. The research provided a feasible method for remanufacturing cost forecast of the engineering machinery hydraulic cylinder, which can be used in setting the remanufactured parts prices in many situations.
\end{abstract}

Keywords: remanufacturing; engineering machinery; cylinder; cost estimation; the neural network

\section{Introduction}

Remanufacturing is a process of restoring a used product to like-new condition by rebuilding it, replacing certain components, and providing a warranty for the remanufactured product that is at least as good as the

\author{
Yun Sun ${ }^{2}$ \\ ${ }^{2}$ Beijing Normal University, Zhuhai 519085, China; \\ Macao University of Science and Technology, Macao
}

\author{
Chun-lan Yang ${ }^{1}$ \\ ${ }^{1}$ College of Mechanical Engineering, Guangxi \\ University,
}

Nanning 530004, China

warranty for a new product. Thus, remanufactured products meet the demand of consumers who desire low-priced, environmentally friendly products that have like-new quality [1]. Remanufacturing has numerous benefits for the original equipment manufacturers (OEMs) [2]. These include savings in labor, material and energy costs; shorter production lead times; balanced production lines; new market development opportunities, and a positive, socially concerned image[3]. The profitability of remanufacturing has resulted in accelerated growth in the remanufacturing industry.

As China pays more attention to the remanufacturing and reengineering, companies are increasingly committed to the development of such. However, remanufacturing industry development in China still faces many difficulties and challenges. Whether recycled old pieces be remanufactured at the technical level and be beneficial for the economic concern to the remanufacturing enterprises lay on the technical and economical evaluation correctness. The remanufacturing cost of estimation, the accuracy of the estimates and the time it takes to produce cost estimates are important factors influencing the ability of remanufacturing organizations that develop, manufacture, sell and support products to survive in today's highly competitive markets [4].Therefore, the evaluations of technic and economic are the key parts and prerequisite conditions for remanufacturing enterprises to create economic benefit. 
The hydraulic cylinder is the vital part to manufacture loaders and excavators. Due to the uncertainty of the working environment of the mechanical engineering, the differences in failure mode and failure degree of the hydraulic cylinder are very huge. Therefore, the requirements for the repairing technology and solution are very complex. This situation makes the remanufacturing cost forecast becomes very difficult due to the high uncertainty. Artificial neural network has widespread application in the field of product cost prediction because of the highly nonlinearity and strong ability of fuzzy reasoning [5-8]. However, this technology has not being widely applied in remanufacturing cost prediction.

The increasing intensity of cost based competition motivated a significant research effort aimed at developing tools that support the cost estimating analysis. Most cost estimates are based on information about the product, the materials it is made of and the production processes required for its manufacturing [4]. The approach base on the remanufacturing processes is applicable for cost estimations in other areas of remanufacturing in which a historical cost database can be constructed and used to design and teach the neural network.

\section{The analysis of cylinder remanufacturing process}

Using one engineering machinery remanufacturing enterprise's actual production process as an example, we can scratch out the technological process of producing cylinder, as shown in figure 1. There are five major steps in the process [9]: (1) the recycling of old pieces; (2) disassembling; (3) cleaning; (4) detection; and (5) technology of repairing.

Recycling is the first step of remanufacturing reverse logistics. What happens here is to take back the old pieces from the customer. When the products cannot function normally or scraped, it would be taken back to the remanufacturing center by the specialists or taken back by the customer himself. During the taken back process, it is necessary to confirm the source of old pieces, the arrangement of transportation, and storage of old pieces.
A preliminary classification is needed when necessary. The source of recycling parts, quality, quantity, and so on will directly affect the production of remanufacturing. Currently the old parts of cylinder mainly come from guaranteed old parts dealer, and the average reusable rate is above $60 \%$.

The second step is the disassembling, which refers to break down the worn-out product into parts and to prevent the damage of parts in the disassembling process. Scientific disassembling technology can effectively ensure the quality and geometric accuracy of reproducing parts, reduce the remanufacturing cycle and cost significantly, and improve the quality of reproducing products. Since the hydraulic cylinder has good sealing capability and its parts work in high precision formation, so it really needs to be handled in a precise way during dismantling to avoid the secondary damage, which may increase the cost and difficulties of repairing.

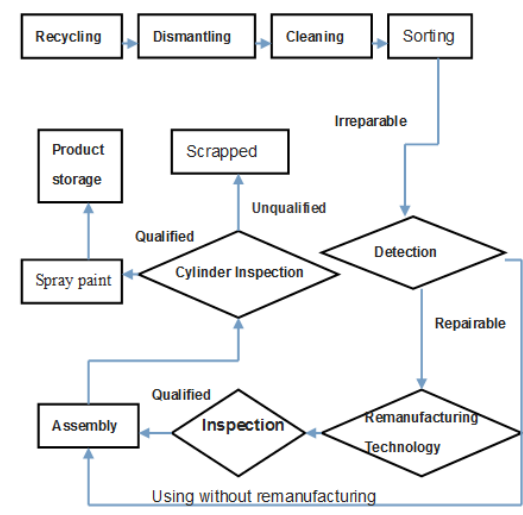

Fig.1 Hydraulic cylinder remanufacturing process

Cleaning is the third step. Surface cleaning is important for the testing of the surface properties, dimensional accuracy, surface roughness, surface geometry precision, and surface adhesive and abrasive. The quality of the surface cleaning directly affects the quality of surface inspection, surface analysis, remanufacturing, and assembly, and further influence the quality of the remanufacturing products. Cleaning process includes three stages: the first is the cleaning of old parts before check in or before dismantling; the second is the cleaning to remove paint and mud after dismantling; the third is the cleaning before sorting and assembling. 
The forth step come after the cleaning is the detection stage. This step includes using various testing tools to determine the surface of the parts geometric parameters, function, etc. so people can determine whether the dismantled parts meet the requirements of the assembly process. It is necessary to test both the appearance and internal quality in detail about the damage of scraped parts, and then based on the test results to make the comprehensive evaluation of remanufacturing and to decide the weather to use the parts. Detection can not only determine the use of scraped parts, but also help to determine the repair plan for the remanufacturing. Detection directly affects the consistency of remanufacturing production cost and the quality. The main detection techniques of remanufacturing include penetration testing, magnetic particle testing, ultrasonic testing, eddy current and magnetic memory testing, electrochemical detection, phased-array ultrasonic testing, etc. Nondestructive testing technology is used for testing the parts internal and surface defects, such as technical conditions, wear, deformation degree and so on.

The fifth step is the repairing. Main technologies in this step include: brush plating, thermal spraying, micro pulse resistance welding, laser cladding, etc. Electro-brush plating technology is used to recover the dysfunctional inner hole and shaft parts that due to the abrasive. By electro-brush plating layers, the dysfunctional parts can regain its physical dimension and enhance its abrasive resistance and even can meet or exceed the performance indicators of new products. Thermal spraying makes the use of some heat sources, such as voltaic arc, plasma arc, or flame to melted or partially melted the metal or nonmetal particles. And then with the help of the combustion flame powder or other high-pressured air stream, people can atomize the melted particles and apply these atomized particles to the primary structure for coating, thus the coating can function as part of the primary structure. Thermal spraying technology is mainly used to recover surface size and performance, which are due to the wear and corrosion, of the wasted parts. This technology can also improve the wear and corrosion resistance of parts through the selection of appropriate coating materials. Micro-pulse resistance welding technology uses the electrical resistance to create high temperature and weld the repairing materials to the primary structure. During this process, the working area's overall temperature rise only by very small amount, so it is a kind of "cold welding" technology. And thus this technology is suitable for repairing the small defect parts, especially suitable for the parts' with heat-treated, different property, high alloy content, or high roughness surface to have micro-amount and high precision repairing. Laser cladding technology places the chosen different filling methods to coat the material on objects' surface, and melts it by the laser radiation to form a low dilute ability and metallurgical bonding thin layer.

The above process analysis indicates that the severity of failure, the original quality of old parts, the repairing technology, the technical requirements and so on are all the important factors in influencing the cylinder remanufacturing cost.

\section{The features of hydraulic cylinder remanufacturing production cost}

\subsection{The relationship between features and the cost of production}

We determine the features that affect the cylinder remanufacturing production cost based on the cylinder remanufacturing process and denote the features with $x_{i}$.

The relationship between the production costs and $x_{i}$ are shown in table 1 , included both the quantitative and qualitative indicators.

\subsection{Features and cost data normalization process}

Since the result of the back-propagation neural network input and output are both between $[0,1]$, it is necessary to normalize the data, valuing between $[0,1]$. Then we transform the qualitative indicators to quantitative indicators, such as transforming the degrees 
of disassembling, cleaning, testing and assembly index into three levels. Then we denote $x_{i}$ 's maximum value as $x_{i \max }$ and minimum value as $x_{i \min }$. The corresponded normalized features factors $\bar{x}_{i}$ can be proceed with the following method.
When features become larger (smaller) and the cost becomes lower (higher), the normalization formula is: $\bar{x}_{i}=\left(x_{i}-x_{i \min }\right) /\left(x_{i \max }-x_{i \min }\right)$.

When features become smaller (larger) and the cost becomes lower (higher), the normalization formula is: $\bar{x}_{i}=\left(x_{i \max }-x_{i}\right) /\left(x_{i \max }-x_{i \min }\right)$.

Tab. 1 The relationship between features and costs

\begin{tabular}{|c|l|l|}
\hline \multicolumn{1}{|c|}{ Features } & \multicolumn{1}{c|}{ Effects on costs } \\
\hline$x_{1}$ & Old parts recycle grades & The lower the grade, the more complicate on repairing, thus the higher the costs \\
\hline$x_{2}$ & Old parts recycle prices & The higher the recycle price, the higher the cost \\
\hline$x_{3}$ & The difficulty of disassembly & The more severe of failure level, the more complex of repairing, the higher the costs \\
\hline$x_{4}$ & The difficulty of cleaning & The more severe of failure level, the more complex of repairing, the higher the costs \\
\hline$x_{5}$ & The difficulty of inspection & The more complex of the inspection, the higher the costs \\
\hline$x_{6}$ & The complicity of repairing technology & The more severe of failure level, the more complex of repairing, the higher the costs \\
\hline$x_{7}$ & Material costs/(Yuan) & The more severe of failure level, the more complex of repairing, the higher the costs \\
\hline$x_{8}$ & Utility expenses/(Yuan) & The more severe of failure level, the more complex of repairing, the higher the costs \\
\hline$x_{9}$ & Equipment costs/(Yuan) & The more severe of failure level, the more complex of repairing, the higher the costs \\
\hline$x_{10}$ & The difficulty of assembling & The more complex of the structure, the higher the cost \\
\hline$x_{11}$ & Weight(kilogram) & The heavier the weight, the higher the cost \\
\hline
\end{tabular}

[10].

\section{The establishment of the BP Neural Network Prediction Model}

Based on the relationship between features and the cost of production, we set the old parts recycle grades, old parts recycle prices, difficulty of disassembly, difficulty of cleaning, difficulty of inspection, raw material cost/(yuan), the utility expenses/(yuan), equipment cost/(yuan), the difficulty of assembling, and weight these 11 features as the input of network and set the remanufacturing production cost as the output in establishing the BP neural network structure. This is a single hidden layer BP network, the number of hidden layer nodes initially has been set at 15 and one for the output layer. The number of neurons in the hidden layer was found by trial and error since the number of hidden units per layer is more of an art than a science

\section{Applications}

\subsection{Sample normalization process}

Normalized features and cost data are shown in tab. 2. Using the first 15 data of samples cylinder in tab. 2 as the training sample, the 16 th as the prediction test sample. Since the cost belongs to confidential data, so tab.2 only provides normalized processing of data. Using this method to forecast cost, get the prediction of manufacturing cost (Yuan) based on the known data normalized reduction.

\subsection{Network testing}

We test the BP network repeatedly by using MATLA and finish when the testing number reaches 3000 and error precision reaches value of 0.0001 , this 
indicates that network structure parameters of the weights and thresholds are set.

After 1,365 times of running, the BP network's testing error sum of squares has a value $e<0.0001$, the mode converges.

\subsection{Results interpretation}

Tab. 3 presents the actual cost and prediction cost of sample cylinder. The maximum different between the predicted value and the actual value is $5.16 \%$. Using 16 groups of samples in table 2 to test the network, the prediction cost of BP model is 1.066 while the actual cost is 1.046. The different between the actual cost and the BP model's prediction error is only $1.9 \%$, far less than $5.16 \%$. Through normalized reduction cost data, we found that the difference between predicted cost and actual cost is only RMB 126 , which is well within the acceptance range in the actual production.

\section{Conclusion}

The neural network based cost estimation model is simple, consistent and accurate. The cost estimates are not based on any subjective judgmental input and therefore different users of the model should consistently produce the same cost estimates. Future applications should concentrate on the design of the interface between the neural network model and the remanufacturing economy evaluation system to provide a link and automatic cost estimates for the users.

\section{Acknowledgment}

The research was supported by Guangxi University Science Research, Project XBZ140447.

\section{References}

[1] Cheng-Han $\mathrm{Wu}$, "OEM product design in a price competition with remanufactured product," Omega ,vol.41 ,2013,pp.287-298.

[2] Geyer, R., Van Wassenhove, and L. N., \& Atasu, A. , "The economics of remanufacturing under limited component durability and finite life cycles on remanufacturing profit,"Management Science, vol. 53(1), pp. 88-100.

[3] Guide Jr, and V. D. R. Jr, "Production planning and control for remanufacturing:Industry practice and research needs," Journal of Operations Management, vol.18(4), 2000,pp.467-468

[4] Serra Caner Bulmus, Stuart X. Zhu, and Ruud Teunter, "Competition for cores in remanufacturing," European Journal of Operational Research, vol.233,2014,pp.105-113.

[5] Avraham Shtub and Ronen Versano, "Estimating the cost of steel pipe bending, a comparison between neural networks and regression analysis," International Journal of Production Economics, vol.62 ,1999,pp.201-207.

[6] Sergio Cavalieri, Paolo Maccarrone, and Roberto Pinto, "Parametric vs. neural network models for the estimation of production costs: A case study in the automotive industry," International Journal of Production Economics, vol.91(2),2004,pp.165-177.

[7] A. Shtub and Y. Zimmerman, "A neural-network-based approachfor estimating the cost of assembly systems," International Journal of Production Economics, vol.32,1993,pp.189-207.

[8] Y.F. Zhang, J.Y.H. Fuh, and W.T. Chan. "Feature-based cost estimation for packaging products using neural networks," Computers in Industry,vol.32 ,1996,pp.95-113.

[9] Surendra M. Gupta and Charles R McLean. "Disassemble of products," Computers ind. Engng,vol. 31,1996,pp.225 -228.

[10] N. Karayiannis, "Theory of Backpropagation Neural Network,",International Joint Conference on Neural Networks,Baltimore, MD, 1992. 
Tab.2 Study and forecasting samples (normalized data )

\begin{tabular}{|c|c|c|c|c|c|c|c|c|c|c|c|c|}
\hline \multirow[t]{2}{*}{ Number } & \multicolumn{11}{|c|}{ Features } & \multirow{2}{*}{ cost } \\
\hline & $\bar{x}_{1}$ & $\bar{x}_{2}$ & $\bar{x}_{3}$ & $\bar{x}_{4}$ & $\bar{x}_{5}$ & $\bar{x}_{6}$ & $\bar{x}_{7}$ & $\bar{x}_{8}$ & $\bar{x}_{9}$ & $\bar{x}_{10}$ & $\bar{x}_{11}$ & \\
\hline NO.1 & 0.500 & 0.361 & 1.000 & 0.500 & 0.500 & 0.500 & 0.350 & 1.000 & 1.000 & 0.500 & 0.361 & 1.377 \\
\hline NO. 2 & 0.500 & 0.337 & 1.000 & 0.500 & 0.500 & 0.500 & 0.300 & 1.000 & 1.000 & 0.500 & 0.337 & 1.448 \\
\hline NO.3 & 0.500 & 0.516 & 1.000 & 0.500 & 0.500 & 1.000 & 0.400 & 1.000 & 1.000 & 0.500 & 0.516 & 1.179 \\
\hline NO.4 & 0.500 & 0.692 & 1.000 & 0.500 & 0.500 & 0.500 & 0.500 & 1.000 & 1.000 & 0.500 & 0.692 & 0.746 \\
\hline NO.5 & 0.500 & 1.000 & 1.000 & 0.500 & 0.500 & 0.500 & 1.000 & 1.000 & 1.000 & 0.500 & 1.000 & 0.319 \\
\hline NO.6 & 0.500 & 0.981 & 1.000 & 0.500 & 0.500 & 0.500 & 1.000 & 1.000 & 1.000 & 0.500 & 0.981 & 0.319 \\
\hline NO.7 & 0.500 & 0.673 & 1.000 & 0.500 & 0.500 & 0.500 & 1.000 & 1.000 & 1.000 & 0.500 & 0.673 & 0.999 \\
\hline NO.8 & 1.000 & 0.000 & 0.500 & 0.500 & 0.500 & 0.500 & 0.500 & 1.000 & 1.000 & 0.500 & 0.000 & 2.543 \\
\hline NO.9 & 0.500 & 0.758 & 1.000 & 0.500 & 0.500 & 0.500 & 1.000 & 1.000 & 1.000 & 0.500 & 0.758 & 0.826 \\
\hline NO.10 & 0.000 & 0.937 & 0.000 & 0.500 & 0.000 & 0.000 & 0.700 & 0.000 & 0.000 & 0.000 & 0.937 & 0.435 \\
\hline NO.11 & 0.000 & 0.937 & 0.000 & 0.500 & 0.000 & 0.000 & 0.700 & 0.000 & 0.000 & 0.000 & 0.937 & 0.434 \\
\hline NO.12 & 0.000 & 0.431 & 0.000 & 0.500 & 0.000 & 0.000 & 0.500 & 0.000 & 0.000 & 0.000 & 0.431 & 1.244 \\
\hline NO.13 & 0.000 & 0.422 & 0.000 & 0.500 & 0.000 & 0.000 & 0.500 & 0.000 & 0.000 & 0.000 & 0.422 & 1.183 \\
\hline NO.14 & 0.500 & 0.361 & 0.500 & 0.500 & 0.500 & 1.000 & 1.000 & 1.000 & 0.667 & 0.500 & 0.361 & 1.326 \\
\hline NO. 15 & 0.500 & 0.465 & 0.500 & 0.500 & 0.500 & 1.000 & 1.000 & 1.000 & 0.667 & 0.500 & 0.465 & 1.110 \\
\hline NO.16 & 0.500 & 0.494 & 0.500 & 0.500 & 0.500 & 1.000 & 1.000 & 1.000 & 0.667 & 0.500 & 0.494 & Predicted cost \\
\hline
\end{tabular}

Tab.3 Actual cost and predicted cost

\begin{tabular}{|c|c|c|c|c|c|}
\hline Numbers & NO.1 & NO.2 & NO.3 & NO.4 & NO.5 \\
\hline Predicted & 1.377 & 1.448 & 1.179 & 0.746 & 0.268 \\
\hline Actual & 1.365 & 1.434 & 1.197 & 0.756 & 0.282 \\
\hline Error/\% & 0.89 & 1.00 & 1.55 & 1.40 & 5.16 \\
\hline Number & NO.6 & NO.7 & NO.8 & NO.9 & NO.10 \\
\hline Predicted & 0.319 & 0.999 & 2.543 & 0.826 & 0.435 \\
\hline Actual & 0.311 & 0.988 & 2.546 & 0.828 & 0.432 \\
\hline Error/\% & 2.65 & 1.08 & 0.13 & 0.21 & 0.63 \\
\hline Number & NO.11 & NO.12 & NO.13 & NO.14 & NO.15 \\
\hline Predicted & 0.434 & 1.244 & 1.183 & 1.326 & 1.110 \\
\hline Actual & 0.432 & 1.248 & 1.182 & 1.315 & 1.122 \\
\hline Error/\% & 0.40 & 0.32 & 0.09 & 0.85 & 1.12 \\
\hline
\end{tabular}

